\title{
Relationships between valence towards wildlife and wildlife value orientations
}

\begin{abstract}
Research to understand human responses to wildlife and wildlife issues has predominantly focused on cognitions. Yet, as emotions are basic human responses to wildlife, emotions are important too. Integrating cognition and emotion concepts could foster the overall understanding of human-wildlife relationships. This study tested the relationships between valence (the pleasant-unpleasant dimension of emotion) regarding wildlife and wildlife value orientations (patterns of basic cognitions in the context of wildlife). Also, this study estimated the additional predictive potential of emotion next to cognition for the acceptability of lethal control and support for wildlife conservation. Analyses showed that valence was associated with wildlife value orientations. Valence had additional explanatory value next to cognition for conservation support. Valence, however, did not have additional predictive potential for acceptability of lethal control. Based on these findings, we recommend integrating cognition and emotion measurements in future research to understand human responses to wildlife issues. Also, wildlife managers could take the emotion of stakeholders into account in their communication and decision-making processes.
\end{abstract}

Keyword: Cognition; Emotion; Wildlife conservation support; Acceptability of lethal control 